\title{
Prevalence of noncardiac findings on low dose 64-slice computed tomography used for attenuation correction in myocardial perfusion imaging with SPECT
}

\author{
Lars Husmann - Fuminari Tatsugami - Ursina Aepli · Bernhard A. Herzog • \\ Ines Valenta · Patrick Veit-Haibach · Ronny R. Buechel · Aju P. Pazhenkottil • \\ Oliver Gaemperli · Nina Burkhard · Christophe A. Wyss · Philipp A. Kaufmann
}

Received: 17 May 2009/Accepted: 28 July 2009/Published online: 7 August 2009

(C) Springer Science+Business Media, B.V. 2009

\begin{abstract}
Electrocardiogram (ECG)-triggered, low dose computed tomography (CT) is increasingly used for attenuation correction in myocardial perfusion imaging (MPI) with SPECT. The purpose of the study was to assess the prevalence of relevant noncardiac findings in the field-of-view of such attenuation correction CT scans. Five hundred and eighty-two consecutive patients (211 female, 371 male; mean age: $64 \pm 11$ years; BMI: $27.7 \pm 5.3 \mathrm{~kg} / \mathrm{m}^{2}$ ) underwent 64-slice, ECG-triggered CT scanning for attenuation correction of MPI with SPECT. Relevant findings were defined as abnormalities that required clinical or radiological follow-up. Noncardiac findings were detected in 400 patients (68.7\%). In 196 patients $(33.7 \%) 226$ relevant findings were detected. Findings included noncalcified pulmonary nodules
\end{abstract}

Lars Husmann and Fuminari Tatsugami contributed equally to this work.

L. Husmann · F. Tatsugami · U. Aepli .

B. A. Herzog · I. Valenta P P. Veit-Haibach .

R. R. Buechel · A. P. Pazhenkottil - O. Gaemperli

N. Burkhard - C. A. Wyss · P. A. Kaufmann ( $\square)$

Cardiovascular Center, Nuclear Cardiology, Cardiac

Imaging, University Hospital Zurich, Ramistrasse 100,

8091 Zurich, Switzerland

e-mail:pak@usz.ch

P. A. Kaufmann

Zurich Center for Integrative Human Physiology,

University of Zurich, Zurich, Switzerland $(n=156)$, interstitial lung disease $(n=6)$, pleural effusion $(n=20)$, pneumonia $(n=1)$, aortic aneurysm $(n=5)$, aortic dissection $(n=4)$, enlarged mediastinal lymph nodes $(n=5)$, mediastinal tumor $(n=3)$, breast abnormalities $(n=3)$, liver cirrhosis $(n=5)$, liver mass $(n=5)$, ascites $(n=5)$, splenomegaly $(n=2)$, renal mass $(n=1)$, hydronephrosis $(n=1)$, adrenal mass $(n=3)$, and bone metastasis $(n=1)$. As low dose 64-slice CT scans used for attenuation correction in MPI with SPECT reveal a high prevalence of noncardiac pathologic findings with potential clinical relevance, a systematic review of the CT scans appears mandatory.

Keywords Incidental findings .

Computed tomography - Attenuation correction . Myocardial perfusion imaging - SPECT

$\begin{array}{ll}\text { Abbreviations } \\ \text { CAD } & \text { Coronary artery disease } \\ \text { CT } & \text { Computed tomography } \\ \text { MPI } & \text { Myocardial perfusion imaging } \\ \text { SPECT } & \text { Single-photon emission computed tomog- } \\ & \text { raphy }\end{array}$

\section{Introduction}

Recently, electrocardiogram (ECG)-triggered, low dose 64-slice computed tomography (CT) has been 
introduced and established for attenuation correction in myocardial perfusion imaging (MPI) with singlephoton emission computed tomography (SPECT) (Fig. 1) [1-6]. The CT data used for attenuation correction is acquired with a large field-of-view, thus including large portions of the great vessels, the lungs, the chest wall, the mediastinum, the vertebra, and the upper abdomen. Although these CT scans are performed with low radiation dose and without contrast material, it can be assumed that incidental relevant or non-relevant noncardiac findings might be found if CT data is adequately reconstructed and reviewed. To our knowledge, no information currently exists on the rate of incidental findings in patients undergoing low dose CT scans for attenuation correction in MPI. Therefore, the purpose of our study was to assess the prevalence of relevant and non-relevant noncardiac findings in the field-of-view of attenuation correction CT scans used for MPI with SPECT.

\section{Methods}

Patients

We prospectively enrolled 582 consecutive patients (211 female, 371 male), who underwent low dose CT scanning of the heart used for attenuation correction of MPI with SPECT in a time period between February, 2007, and February, 2008. MPI was performed for evaluation of known or suspected coronary artery disease (CAD); in $10 \%$ of patients MPI was part of preoperative evaluation before noncardiac surgery. The study protocol was approved by the institutional review board and written informed consent was obtained.
CT scanning

All CT scans for attenuation correction were performed with a 64-slice CT on a LightSpeed VCT XT scanner (GE Healthcare, Milwaukee, WI, USA) using prospective ECG-triggering ( $70 \%$ of the R-R interval) and the following scanning parameters: gantry rotation time $350 \mathrm{~ms}, 2.5-\mathrm{mm}$ section thickness, $120 \mathrm{kV}$ tube voltage, $200 \mathrm{~mA}$ effective tube-current. Scanning was performed at breath-hold in inspiration with elevated arms from below the tracheal bifurcation to the diaphragm. CT images were reconstructed at a $2.5-\mathrm{mm}$ section thickness by using a reconstruction algorithm with a $512 \times 512$ matrix and a fullchest-size-adapted field-of-view of $50 \times 50 \mathrm{~cm}$. Reconstructed images were transferred to the external workstation for further analysis (AW 4.4, GE Healthcare).

Image analysis

One board-certified radiologist, blinded to the patient's history, reviewed all examinations for noncardiac findings using standard soft tissue (window width W400/level L40 Houndsfield Units), lung (W1600/L600), and bone window (W1700/L250) settings. All noncardiac findings were recorded and rated as relevant, when further clinical or radiological work-up or follow-up was deemed appropriate by this experienced radiologist, similar to previous reports [7-9]. Equivocal findings were categorized as relevant or non-relevant according to the following criteria:

Noncalcified pulmonary nodules larger than $4 \mathrm{~mm}$ were considered relevant and radiological follow-up was recommended [10]. Noncalcified pulmonary
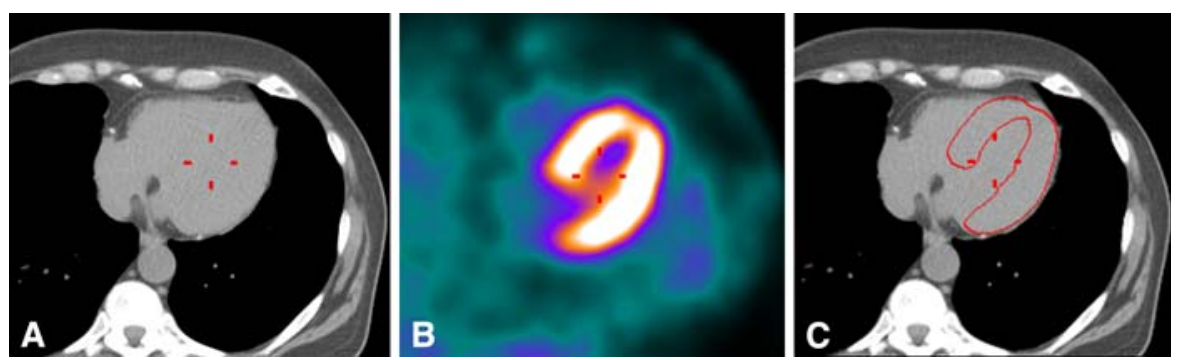

Fig. 1 Axial CT- (a) and SPECT-images (b) are fused (c), for attenuation correction in myocardial perfusion imaging with SPECT. This method is routinely performed in clinical practice to compensate for possible attenuation artefacts in myocardial perfusion images 
nodules larger than $10 \mathrm{~mm}$ and nodules smaller than $10 \mathrm{~mm}$, but with irregular margination were considered potentially malignant and further work-up was suggested. Mediastinal lymph nodes were described as enlarged if their short axis measured more than $10 \mathrm{~mm}$ [11]. Aortic aneurysm was defined as a dilation equalling or exceeding twice the normal diameter. Dissections were suspected if they had a medial displacement of calcifications in the aorta [12].

A clinical follow-up of all relevant findings was performed in August 2008 by reviewing of individual medical records.

Statistical analysis

Quantitative variables were expressed as mean \pm standard deviation and categorical variables as frequencies, or percentages, using SPSS software (SPSS 15.0, Chicago, ILL, USA).

\section{Results}

CT for attenuation correction was successfully performed in all 582 patients. The patients mean age was $64 \pm 11$ years (range 30-89 years) and their body mass index was $27.7 \pm 5.3 \mathrm{~kg} / \mathrm{m}^{2}$ (range $15-54$ $\mathrm{kg} / \mathrm{m}^{2}$ ). In patients without previous bypass surgery or stent placement (390 patients, 67.0\%) the mean coronary artery calcium scoring was $564.6 \pm 942.4$ (range 0-6,728). Further clinical characteristics of the patient population are summarized in Table 1 .

\section{Noncardiac findings}

A total of 832 findings were detected in 400 of the 582 patients $(68.7 \%)$. Two hundred and twenty-six findings were classified as having potential clinical relevance, $196(33.7 \%)$ patients had at least one relevant finding (16 patients had two, 5 patients had three, and 1 patient had five relevant findings).

One hundred and fifty-six patients $(26.8 \%)$ had one or more noncalcified pulmonary nodules, rated as relevant findings, as a follow-up was recommended.

CT revealed an abnormality in the mediastinum in eight patients (1.3\%), mediastinal lymph nodes adenopathy in five, retrosternal mass in two, and a tumor in the middle mediastinum in one patient. Nine
Table 1 Clinical characteristics of the patients

\begin{tabular}{lc}
\hline & $n(\%)$ \\
\hline Symptoms & \\
Chest pain & $195(34)$ \\
Dyspnea & $50(9)$ \\
Combination of both & $53(9)$ \\
History of CAD & \\
Previous PCI & $245(42)$ \\
Previous bypass surgery & $55(9)$ \\
Previous myocardial infarction & $107(18)$ \\
Coronary risk factors & \\
Hypertension & $329(57)$ \\
Smoking & $235(40)$ \\
Dyslipidemia & $217(37)$ \\
Positive family history & $159(27)$ \\
Diabetes & $112(19)$ \\
\hline
\end{tabular}

$P C I$ percutaneous coronary intervention, $C A D$ coronary artery disease

findings in the ascending and descending aorta were documented in eight $(1.3 \%)$ patients, five aneurysms and four dissections.

In patients with liver cirrhosis $(n=5)$ and splenomegaly $(n=2)$ clinical or radiological follow-up was recommended. In patients with suspected liver tumor $(n=5)$, renal tumor $(n=1)$, adrenal tumor $(n=3)$, hydronephrosis $(n=1)$, or ascites $(n=5)$ further investigation was suggested.

Eleven (1.9\%) patients had bone abnormalities. Eight had vertebral hemangioma, and 2 had rib fractures. One patient had vertebral osteolysis suspicious for metastasis.

The total number of incidental noncardiac findings and of relevant findings are summarized in Table 2, examples are shown in Fig. 2.

Follow-up of relevant findings

During a follow-up period of 6-18 months, 129 of 226 relevant findings were lost to follow-up (57.1\%). Seventy-four findings were already known prior to CT scanning (32.7\%). Three findings (1.3\%) were not confirmed on follow-up, i.e. one kidney tumor, and two pulmonary nodules $<10 \mathrm{~mm}$. Adequate detection of previously unknown relevant findings was confirmed in 20 cases $(8.8 \%$ ) (Table 2), interestingly, 3 of 5 lung nodules $>10 \mathrm{~mm}$ turned out to be a newly detected bronchial carcinoma. 


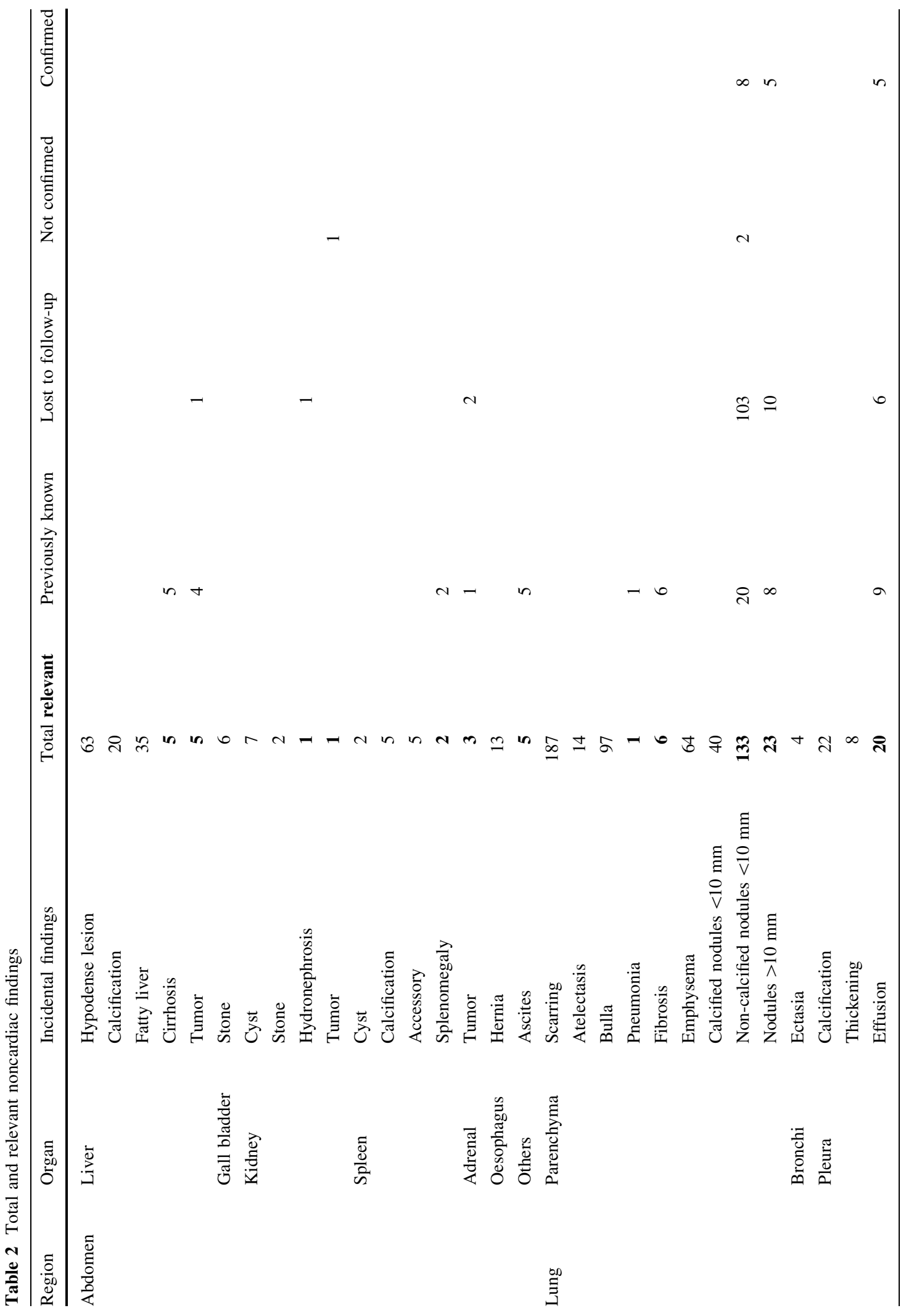




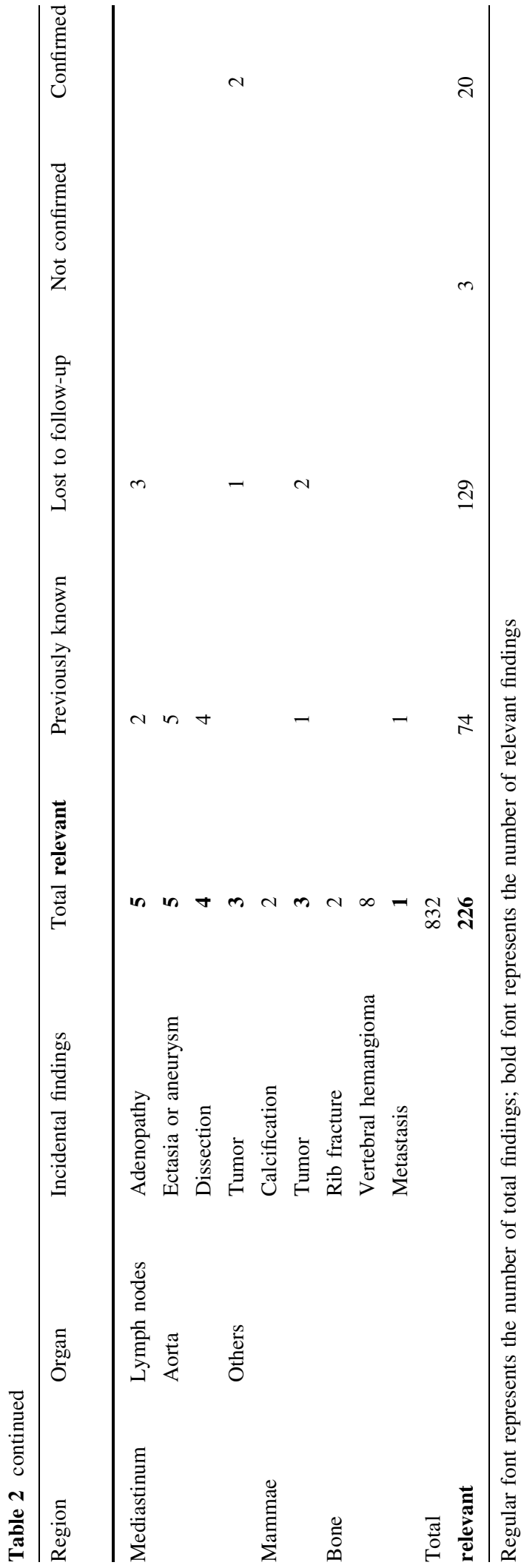

\section{Discussion}

This study demonstrates for the first time that the prevalence of non-relevant and clinically relevant noncardiac findings is high in 64-slice CT scans used for attenuation correction in MPI with SPECT.

Several previous studies have assessed the frequency of incidental findings in the context of various types of cardiac CT examinations [7-9, 13, 14], most of them involved electron beam CT or full dose coronary CT angiography. Our study revealed a higher prevalence of incidental $(68.7 \%)$ and clinically relevant incidental findings $(33.7 \%)$ as compared to previous electron-beam CT studies for calcium scoring [7-9]. In the latter reports, the detection rates for abnormalities ranged from 20 to $53 \%$ for all findings and from 4.2 to $9.3 \%$ for relevant findings. However, in those studies, electron-beam CT images were focused on the heart and a smaller field of view was used $(26-35 \mathrm{~cm})$ compared to our study $(50 \mathrm{~cm})$. Furthermore, electron-beam CT is known to have a lower spatial resolution as compared to 64-slice CT. Both, the smaller field-of-view and the lower spatial resolution might explain the lower prevalence of noncardiac findings in electron-beam CT studies compared to our study.

In cardiac multidetector CT examination using a large field-of-view including the chest wall, Onuma et al. [13] found in $58.1 \%$ of the patients at least one noncardiac finding, and in $22.7 \%$ of patients CT follow-up was recommended. Similarly, Goetze et al. [15] described noncardiac findings in 59\% of patients on low-dose single-slice CT scans for MPI attenuation correction, suggesting that this "non-diagnostic" CT portion will likely become more important once higher quality $\mathrm{CT}$ scanners are used. In fact, the prevalence of noncardiac findings in our 64-slice CT study proved to be higher than their results. Especially, the rate of noncalcified nodules smaller than $10 \mathrm{~mm}$ in our study $(22.8 \%)$ was much higher than in previous reports $(9.7 \%)$ [13], in which CT images with relatively thick slices $(5 \mathrm{~mm})$ compared to our study $(2.5 \mathrm{~mm})$ were used, which may have lead to underestimating the prevalence of small size lung nodules.

Gil et al. [14] also examined patients who underwent cardiac CT angiography using a large field of view, and $56.2 \%$ of patients were found to have a relevant noncardiac finding. In that report, the 

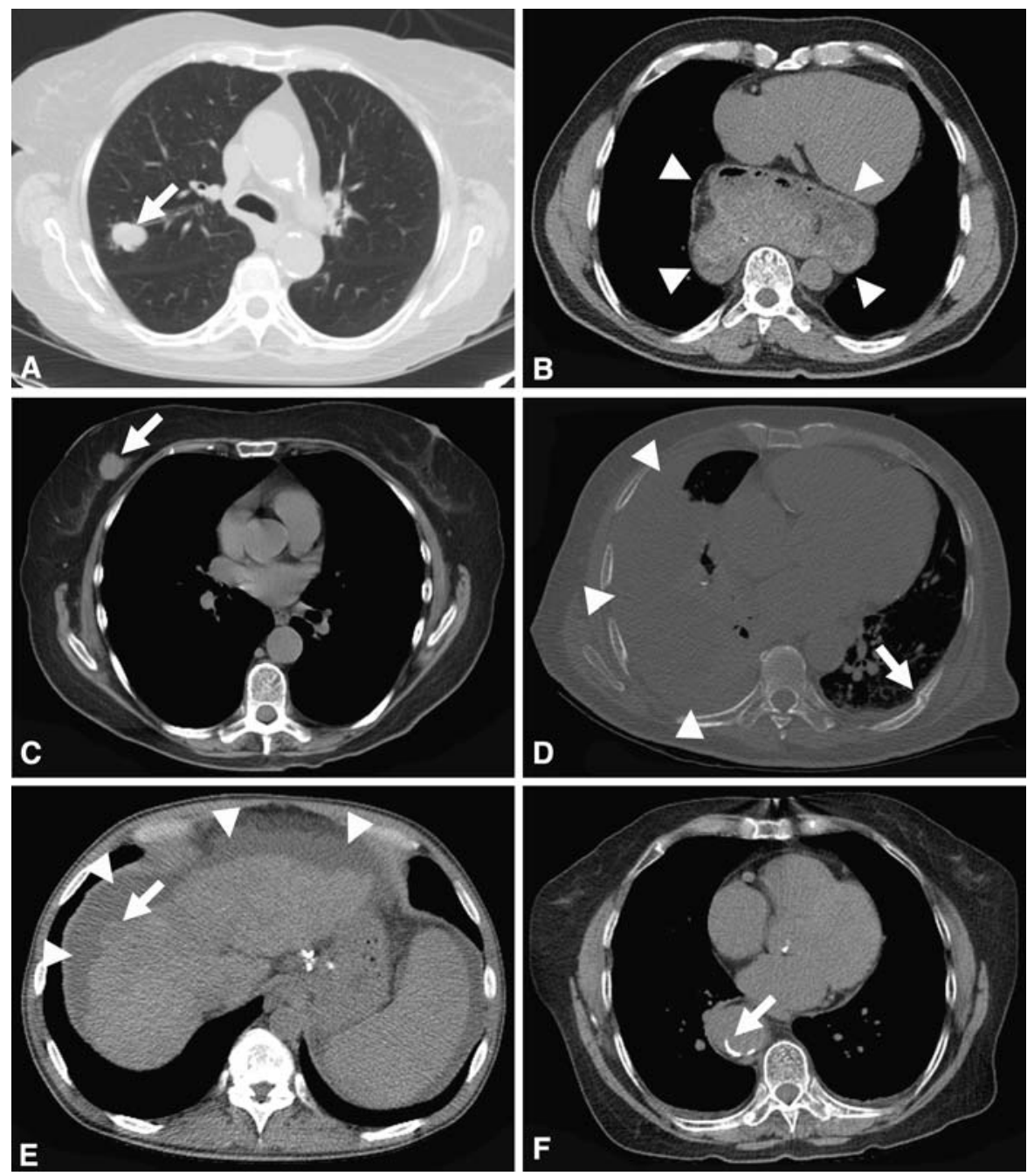

Fig. 2 Incidental noncardiac findings on low-dose 64-slice CT scans used for attenuation correction in MPI with SPECT. a Lung tumor. b Large hiatal hernia. c Breast tumor. d Large

pleural effusion (arrow heads) and dorsal rib fracture (arrow). e Ascites (arrow heads) and liver tumor (arrow). f Aortic dissection

scan volume was determined from the apex of the lungs to the diaphragm including the upper lobes and thyroid, which could explain the higher prevalence of noncardiac relevant findings compared to our study.

\section{Limitations of the study}

The incidence of findings may be influenced by the patient selection as a large proportion of patients referred for preoperative evaluation before surgery due to an existing disease would confer a high prevalence of findings. In our study, however, only
$10 \%$ of patients fall into this category. Certainly, the relatively advanced age of our study population may have favoured the incidence of comorbidity.

Another limitation of this study is that clinical follow-up was not available in all patients with relevant clinical findings.

\section{Conclusions}

In conclusion, low dose 64-slice CT scans used for attenuation correction in MPI with SPECT provide an image quality sufficient to detect a high prevalence of 
noncardiac pathologic findings with potential clinical relevance in a population typically evaluated for CAD. Therefore, a systematic review of the scans appears mandatory.

Acknowledgments The study was supported by a grant from the Swiss National Science Foundation (SNSF-professorship grant No. PP00A-114706) and from the Zurich Center of Integrative Human Physiology.

\section{References}

1. Masood Y, Liu YH, Depuey G et al (2005) Clinical validation of SPECT attenuation correction using x-ray computed tomography-derived attenuation maps: multicenter clinical trial with angiographic correlation. J Nucl Cardiol 12:676-686

2. Fricke E, Fricke H, Weise R et al (2005) Attenuation correction of myocardial SPECT perfusion images with low-dose CT: evaluation of the method by comparison with perfusion PET. J Nucl Med 46:736-744

3. Preuss R, Weise R, Lindner O et al (2008) Optimisation of protocol for low dose CT-derived attenuation correction in myocardial perfusion SPECT imaging. Eur J Nucl Med Mol Imaging 35:1133-1141

4. Schepis T, Gaemperli O, Koepfli P et al (2007) Use of coronary calcium score scans from stand-alone multislice computed tomography for attenuation correction of myocardial perfusion SPECT. Eur J Nucl Med Mol Imaging 34:11-19

5. Singh B, Bateman TM, Case JA et al (2007) Attenuation artifact, attenuation correction, and the future of myocardial perfusion SPECT. J Nucl Cardiol 14:153-164
6. Rivero A, Santana C, Folks RD et al (2006) Attenuation correction reveals gender-related differences in the normal values of transient ischemic dilation index in rest-exercise stress sestamibi myocardial perfusion imaging. J Nucl Cardiol 13:338-344

7. Hunold P, Schmermund A, Seibel RM et al (2001) Prevalence and clinical significance of accidental findings in electron-beam tomographic scans for coronary artery calcification. Eur Heart J 22:1748-1758

8. Horton KM, Post WS, Blumenthal RS et al (2002) Prevalence of significant noncardiac findings on electron-beam computed tomography coronary artery calcium screening examinations. Circulation 106:532-534

9. Schragin JG, Weissfeld JL, Edmundowicz D et al (2004) Non-cardiac findings on coronary electron beam computed tomography scanning. J Thorac Imaging 19:82-86

10. MacMahon H, Austin JH, Gamsu G et al (2005) Guidelines for management of small pulmonary nodules detected on CT scans: a statement from the Fleischner Society. Radiology 237:395-400

11. Lau CL, Harpole DH Jr (2000) Noninvasive clinical staging modalities for lung cancer. Semin Surg Oncol 18: 116-123

12. Sebastia C, Pallisa E, Quiroga S et al (1999) Aortic dissection: diagnosis and follow-up with helical CT. Radiographics 19:45-60 quiz 149-150

13. Onuma Y, Tanabe K, Nakazawa G et al (2006) Noncardiac findings in cardiac imaging with multidetector computed tomography. J Am Coll Cardiol 48:402-406

14. Gil BN, Ran K, Tamar G et al (2007) Prevalence of significant noncardiac findings on coronary multidetector computed tomography angiography in asymptomatic patients. J Comput Assist Tomogr 31:1-4

15. Goetze S, Pannu HK, Wahl RL (2006) Clinically significant abnormal findings on the "nondiagnostic" CT portion of low-amperage-CT attenuation-corrected myocardial perfusion SPECT/CT studies. J Nucl Med 47:1312-1318 